\title{
Strategies for regulating the emotional sphere of the personality in the context of digitalization
}

\author{
Olga Vasilyeva, and Nadezhda Blinova* \\ Southern Federal University, 344006, Rostov-on-Don, Russia
}

\begin{abstract}
The article is devoted to the study of ideas about forgiveness and readiness for it as an important part of the psycho-emotional life and social realization of a person. Individual ideas about forgiveness, a portrait of a forgiving and unforgiving person are presented. The analysis of the results obtained according to the author's questionnaire "Readiness for forgiveness" is given. Three scales-components of readiness for forgiveness are identified: 1) motivation, 2) understanding and acceptance, 3) gratitude. The relationship between readiness for forgiveness and the characteristics of the emotional sphere of a person is described. Correction of semantic strategies is observed when ideas about forgiveness and the process of forgiveness change.
\end{abstract}

\section{Introduction}

At the present stage of social development, due to the acceleration of scientific and technological progress and the rapidly changing conditions of the social environment, the number of negative factors affecting a person is increasing, which contributes to the growth of disorders in the emotional sphere of the personality [1,2]. The phenomenon of forgiveness raises a number of questions that cover a wide range of scientific fields [3-6]. Despite the widespread use of the concept of forgiveness in spoken language, literature, religious and philosophical texts, the understanding of its essence is ambiguous [7-9].

Due to the ambiguity of understanding the essence of forgiveness, when conducting psychological studies of the phenomenon of forgiveness, the difficulties of a terminological and methodological nature arise. In Christianity, forgiveness is closely related to repentance, it is one of the most important conditions for the soul salvation, which, in fact, is the main meaning-forming process for a Christian. Western models, based on an understanding of the offender by mind, highlight the importance of recognizing the moral principles that give rise to the forgiveness, the most well-known is the Enright's model described in his works [10]. There is a number of approaches to forgiveness, which differ both conceptually and technologically [11-13]. Therefore, the formation of deeper adequate idea of forgiveness is an urgent task of education, in particular in the field of psychology, which can promote a change in semantic strategies [14-16] that lead to the transformation of negative experiences, harmonization of both intrapersonal and interpersonal space, spiritual growth. The importance of studying the phenomenon of readiness for forgiveness becomes especially

\footnotetext{
*Corresponding author: blinova_nadia@mail.ru
} 
relevant when identifying the relationship between it and such components of the emotional sphere of a person as emotional intelligence and subjective well-being [17].

\section{Methods}

And the investigation took place in two stages: at the first - the study of ideas about forgiveness, at the second - the definition of the concept of readiness for forgiveness and the degree of its manifestation in the process of life. At the first stage, an organizational-activity game was developed, the purpose of which is to identify and analyze the most pressing individual problems in the field of forgiveness. For the purpose of researching ideas about forgiveness, an author's questionnaire has been developed for researching ideas about forgiveness. At the second stage, a special questionnaire was developed to determine the level of readiness for forgiveness. To study emotional intelligence we used N. Hall's methodology "Emotional Intelligence". To study the level of subjective well-being we used the subjective well-being scale.

In order to find the answers to the questions set in the work, men and women aged from 30 to 45 were examined (total number of 300 people), of which 130 men and 170 women. The average age of the respondents is 38.4 years old.

Research objectives:

1) To analyze the properties of the ideas of forgiveness;

2) To analyze the level of readiness for forgiveness and highlight the subjects declaring low, medium and high readiness for forgiveness;

3) Establish the relationship between the level of readiness for forgiveness and the characteristics of the emotional sphere.

\section{Results}

As a result of the study of ideas about forgiveness, cognitive, emotional and behavioral aspects of forgiveness were identified. When analyzing responses, it was difficult to establish a clear classification due to the responses variability. It should be noted that the respondents were very active when answering the questionnaire. So, when answering the question "What associations do you have with the words forgiveness?" the average number of associations produced by one respondent is 3.2. When answering the question "What are the characteristics of a person who knows how to forgive? the average number of responses per one respondent was 3.4. When answering the question "What are the characteristics of a person who does not know how to forgive?" average number of responses per one respondent was 3.5. When answering the question "What do you think "forgive" means?" the average number of responses per one respondent, was 1.9. This suggests that respondents have a structured view of forgiveness. Individual perceptions of the concept of "forgiveness" are contradictory. On the one hand, forgiveness is associated as acceptance, understanding, repentance, resentment, pain, relief, release, awareness, calmness, goodness, joy, conversation, harmony, peace, reconciliation, healing, on the other hand, forgiveness is associated as work and internal efforts. Summarizing all, the majority of respondents consider forgiveness to be a necessary, but difficult process, requiring internal efforts and changes that bring joy and emotional relief to the forgiving person and contribute to the harmonization of relations between people.

As a part of the investigation of the forgiveness process the respondents were asked: "What do you think it means to "forgive"? All answers can be divided to the following groups. To forgive means:

1. Reconsideration and acceptance of the situation; 
2. To forget and no longer "stir up" the past;

3. Understanding and acceptance of the offender as he is: with all his advantages and disadvantages;

4. Internal self-work to release pain and resentment.

Thus, the question "what, in your opinion, means "to forgive" divided the surveyed group into four subgroups: the first one supposes to forgive means not to think, to forget; 2 nd - to forgive means to understand and accept the offender; 3rd - to forgive means to understand and accept the situation; 4th - to forgive means to be reconciled with oneself.

Respondents put the border between what is "possible to forgive," and what is "impossible to forgive". The actions that get the most significant psychological resistance in the realization of forgiveness include: betrayal, willful damage to life (mostly - murder, various forms of violence), i.e. - traditionally socially rejected qualities;

In the opinion of the subjects, "it is possible to forgive" the deception; rude joke; treason; "What a person repents of"; "What is done not knowingly, not out of malice." Respondents' answers to the survey "What, from your point of view, cannot be forgiven?" both in organizational-activity games and in survey forms, had the following tendencies: it is impossible to forgive premeditated murder $(24 \%)$, betrayal $(29 \%)$, humiliation $(9 \%)$. It should be noted that $38 \%$ of respondents say that everything can be forgiven. The majority of respondents are aware of the following fact: in words, they are willing to forgive a lot, but often in specific situations, the process of forgiveness is delayed for many years, and sometimes for the rest of life, despite the fact that "they are trying to change their views and feelings to the offender".

A similar border is observed among the subjects in the categories "who can be forgiven" and "who cannot be forgiven". A person, who can forgive, according to respondents, has the following characteristics: cheerfulness, discretion, mercy, kindness, simplicity, generosity, a high level of maturity and a broad look on the world. A person who does not know how to forgive is characterized by: isolation, internal aggression, disbelief, distrust, cruelty, rationalism, fearfulness, intransigence, anger, selfishness, vindictiveness. According to respondents, a person who does not know how to forgive has diseases and complaints.

Analysis of answers to the question "What do you do and/or feel when you forgive?" demonstrates the following spectrum of feelings arising from forgiveness: ease or relief, satisfaction, calmness, relaxation, joy, good nature. The respondents note that, having changed their attitude towards the offender, they "feel lightness in their souls". However, there are those who, feeling relief, do not find the opportunity to communicate with the abuser as before.

When analyzing the answers to the question "How often do you feel offended?" Four categories of answers were singled out: three groups of respondents answered this question absolutely unambiguously: either "often" (15\%), or "rarely" $(35 \%)$, or "sometimes" (30\%). Another group $(20 \%)$ of respondents answered this question "quite often", including his rational replacement, such as: "now less than before," "often, but I struggle with it" etc.

When answering the question "Do I need to learn to forgive? If, "No", then why, if, "Yes", then how can this be learned?" the respondents gave rather long answers with an emphasis on the importance of the process of forgiveness in life. To the question "Do I need to learn to forgive?" $99 \%$ of the respondents answered in the affirmative, $3 \%$ of the respondents defined the direction of learning as mastering religious consciousness, in particular the study of the Bible.

When diagnosing the level of readiness for forgiveness, based on the analysis of ideas about forgiveness obtained at the previous stage of the study, a questionnaire consisting of 47 questions was developed and tested as a tool for assessing the readiness for forgiveness. The following scales of readiness for forgiveness are highlighted: "Motivation for forgiveness", "Understanding and accepting" "Gratitude". Theoretical analysis and extensive 
practical experience allow us to assume that gratitude, absence of resentment, discontent, claims to oneself, to parents, to society determine the readiness for forgiveness. For example, we give the results on the scale of "Understanding and Acceptance", which reflects the degree of resentment, dissatisfaction, claims to oneself, to parents, to others and the degree of acceptance of oneself and others. The scale contains both direct, and opposite questions. The criterion for attributing a respondent to a stratum with a low, medium or high readiness for forgiveness was the answers to the questions assigned to the "Understanding and Acceptance" scale:3. I forgive a lot; 6 . I often get offended; 7. I am often dissatisfied with the actions of various government agencies; 26. I have complaints, resentments towards myself; 27. I have complaints, resentments against many people; 28. I have no complaints, resentments to many people; 29. I get offended sometimes; 30. I have no complaints, resentments to myself; 31 . I have no complaints, resentments to my mother; 33 . I can forgive everything; 35. I have complaints, resentments to my father; 36. I have complaints, resentments to my mother;37. I am unhappy with my father;39. I am unhappy with my mother; 40. I have no complaints, resentments to my father; 42. I am unhappy with my fate;43. I forgive unconditionally.

Taking into account the fact that the answer options of the questionnaire assume a fixed form with a scale system from -3 to +3 and the content of the questions, we get an estimate (from -26 to 5), which should be interpreted as follows: the lower the score, the higher readiness for forgiveness. In accordance with the received assessment, the respondents were divided into three groups: with high, medium and low levels of readiness to forgive. The respondents with an assessment of the level of readiness for forgiveness from -26 to -15 made up the group with a high level of readiness for forgiveness $(20 \%)$. The respondents with an assessment of the level of readiness for forgiveness from -15 to -5 made up the group with an average level of readiness for forgiveness (48\%). The respondents with an assessment of the level of readiness for forgiveness from -5 to +5 made up the group with a low level of readiness for forgiveness $(32 \%)$.

We believe that among the main mechanisms for the formation of readiness for forgiveness are a person's worldview, features of his emotional sphere, which in our study were diagnosed by the scale of subjective well-being, "Emotional intelligence" (N. Hall). The technique allows you to identify such indicators as self-motivation, emotional awareness, empathy, managing your emotions, and others. To determine the level of emotional intelligence among respondents, we used N. Hall's methodology. With the help of this technique, indicators were identified for each scale of the questionnaire and an integrative indicator of emotional intelligence was calculated. Three groups were identified with high, medium and low EQ scores.A high level of the integrative indicator EQ was found in $12 \%$ of respondents, an average level - $40 \%$ of respondents and a low level - in $48 \%$ of respondents.

To diagnose the level of emotional well-being, the subjective well-being scale was applied. The final score is an indicator of the presence and depth of the emotional discomfort of the individual, and according to the answers to individual points, it can reveal areas of special tension or conflict. The first group consisted of respondents with a high level of emotional well-being (12\%). Low scores on the subjective well-being (SB) or emotional comfort (EC) scale (1 sten score) indicate the complete emotional well-being of the subject and his denial of serious psychological problems. Such a person, most likely, has a positive self-esteem, is not inclined to complain about various ailments, is optimistic, sociable, confident in his abilities, acts effectively under stress, is not prone to anxiety. The second group is represented by respondents with an average level (48\%). Estimates deviating towards subjective well-being (2-3 sten scores) speak of the emotional comfort of the subject: he does not experience serious emotional problems, he is confident enough with himself, is active, successfully interacts with others, and adequately controls his behavior. We 
conditionally defined the third group as a low level (40\%). Average grades (4-7 sten scores) indicate a low expression of the indicator: persons with such assessments are characterized by moderate subjective well-being, they have no serious problems, but one cannot speak of complete emotional comfort. Assessments deviating in the direction of subjective distress (89 sten scores) are typical for people prone to depression and anxiety, pessimistic, withdrawn, dependent, poorly tolerating stressful situations. Those in our sample were not identified (see Fig. 1).

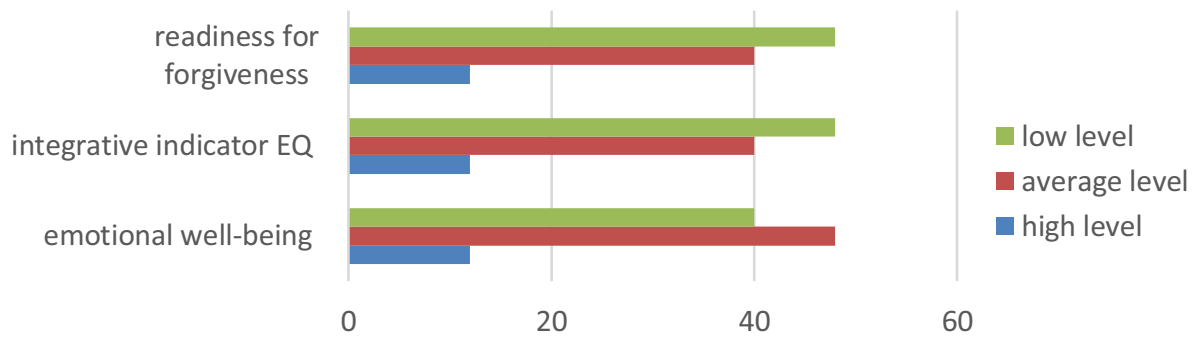

Fig. 1. Groups of respondents with different levels of emotional intelligence, well-being and readiness to forgive.

The study revealed a significant relationship between these indicators and the willingness to forgive. A significant correlation was revealed between the indicators "Level of readiness for forgiveness" and "Emotional awareness" $(\mathrm{r}=-0.481 ; \mathrm{p}=0.014)$, between the indicators "Level of readiness for forgiveness" and "Management of emotions" $(r=-0.549 ; p=0.004)$, between the indicators "The level of readiness for forgiveness" and "Self-motivation" $(\mathrm{r}=$ $0.477 ; p=0.015)$. Indicators of the "Level of readiness for forgiveness" have a significant connection with the "Integrative indicator" of emotional intelligence $(r=-0.487 ; p=0.013)$. There are positive significant correlations between the level of readiness for forgiveness and indicators of such scales of subjective well-being as "Self-assessment of health" $(r=0.431$; $p=0.03)$ and "Degree of satisfaction with everyday life" $(r=0.403 ; p=0.04)$. The more pronounced the feeling of subjective well-being on these scales, the higher the readiness to forgive.

\section{Discussion}

In our opinion, various forms of the organization of group discussions contribute to a deeper study of the socio-psychological aspects of forgiveness [18]. When using information technologies in the process of conducting classes: a video recording of a lesson is placed in an information space, there is an expansion of information capabilities, in particular, the availability of information in temporal and spatial aspects, coverage of a larger number of people. But at the same time, there is a sharp decrease in the quality of feedback, the ability to track changes in the ideas and meanings associated with this information decreases. So, for example, during group work with the topic of forgiveness of parents in a group of 20 students, we used a presentation on this topic and a video with subsequent analysis. During the discussion, there were many different points of view, as a result of the discussion, there were many insights, which allowed the group members to adjust or change their ideas and semantic strategies in relation to this issue.

\section{Conclusions}


The results of this study complement the picture of forgiveness and readiness for forgiveness and can be used in diagnosing problems in the emotional sphere of a person and improving the methods of their prevention through the development of readiness for forgiveness. Therefore, for psychologists, it is important to know about the structure of the forgiveness process, and the factors that help to optimize this process. In this regard, a number of seminars, trainings and organizational-activity games have been developed that allow understanding the features of the phenomenon of forgiveness at the cognitive, emotional and behavioral levels and gaining skills for implementing forgiveness in one's life. Information technologies are an additional tool for the formation of motivation for changes in semantic strategies. Various forms of group work affect the expansion of ideas about forgiveness, help participants adjust their ideas and semantic strategies. Thus, we can say that the phenomenon of forgiveness requires deep attention to its study in connection with its versatility and multiaspect relationship with other psychological phenomena. On one hand, it is relatively simple, since respondents have relatively similar ideas about it. On the other hand, there are tendencies for a deeper and more multifaceted connection of the phenomenon of forgiveness with other characteristics of the emotional sphere.

\section{References}

1. J.N. Hook, E.L. Worthington, S.O. Utsey, D.E. Davis, J.L. Burnette, Counseling and Values 57, 109-124 (2012). doi.org/10.1002/j.2161-007X.2012.00012.

2. G. Nudelman, A. Nadler, Personality and Individual Differences 116, 191-200 (2017). doi.org/10.1016/j.paid.2017.04.048.

3. T.Hashimoto, K. Karasawa, The Japanese Journal of Experimental Social Psychology 51, 104-117 (2012).

4. H.D. Johnson, M.A. Wernli, J.C. LaVoie, The Journal of Genetic Psychology 174, 291315 (2013). doi.org/10.1080/00221325.

5. K. Kaleta, J. Mróz, Annals of Psychology 21, 147-162 (2018). doi.org/10.18290/rpsych.2018.21.2-3.

6. H. Brown, The conditions of forgiveness in recent philosophical thought, A Journey through Forgiveness (Ed. by M.R. Maamri, N. Verbin, E.L. Worthington, Jr. Inter Disciplinary Press, Oxford, 2010).

7. Yu.V. Pechin, Psychological Science and Education psyedu.ru 6 (4), 194-206 (2014).

8. A.R. Beck, L.M. Ruhlmann, J.A. Durtschi, C.C. Brown, The American Journal of Family Therapy 45 (5), 250-263 doi.org/10.1080/01926187.2017. 1365664.

9. S.R. Braithwaite, E.A. Selby, F.D. Fincham, Journal of Family Psychology 25, 551-559 (2011). doi.org/10.1037/a0024526.

10. R. Enright, Forgiveness education with children in areas of violence and poverty, Forgiveness: A Sampling of Research Results, American Psychological Association, (2006) (DC: Office of International Affairs. Reprinted, Washington, 2008)

11. L. Rey, N. Extremera, Terapia Psicológica 34, 103-110 (2016). doi.org/10.4067/S071848082016000200003.

12. C.V. Witvliet, L.R. Luna, E.L. Worthington, Jr., J.A. Frontiers in Psychology 11, 1-12 (2020). doi.org/10.3389/2Ffpsyg.2020.00284.

13. P.Strelan, J.C. Karremans, J. Krieg, British Journal of Social Psychology 56, 161-180 (2017). doi.org/10.1111/bjso.12173. 
14. M.E. McCullough, Beyond Revenge: The Evolution of the Forgiveness Instinct (JosseyBass, San Francisco, 2008).

15. A.S. Chukova, V.V. Gritsenko, Russian Psychological Journal 32 (5), 16-22 (2011).

16. E.L. Worthington, Forgiveness and religion: update and current status, (Worthington)

17. E.L. Davis, D.J. Hook, Van Tongeren D., Gartner A., Jennings D., Greer C., Greer T., A Journey through Forgiveness (Jr. Inter Disciplinary Press, Oxford, 2010).

18. N.N. Blinova, T.N. Kondratieva, O.S. Vasilyeva, E3S Web of Conferences 210, 18085 (2020). https://www.e3s-conferences.org/articles/e3sconf/abs/2020/70/e3sconf_itse

19. O. Vasilyeva, O. Shinkarenko, N. Blinova, Existential tradition: philosophy, psychology, psychotherapy 1 (18), 49-52 (2011). 